\title{
DIFFERENTIABILITY THROUGH CHANGE OF VARIABLES
}

\author{
A. M. BRUCKNER AND C. GOFFMAN ${ }^{1}$
}

\begin{abstract}
A real function $f$ on $[0,1]$ can be transformed by a homeomorphism into a differentiable function with bounded derivative if and only if $f$ is continuous and of bounded variation. This condition does not suffice for $f$ to be transformed into a continuously differentiable function. The additional condition for this to hold is found and the theorem is proved.
\end{abstract}

Let $f$ be a real function defined on the interval $[0,1]$. We show, Theorem 1 , that $f$ can be transformed, by a homeomorphic change of variable, into a differentiable function with bounded derivative, if and only if $f$ is continuous and of bounded variation. This condition does not suffice, however, for $f$ to be transformable into a function with a continuous derivative. For that, it is necessary and sufficient that $f$ satisfy an additional condition. We discuss this condition and then state and prove the resulting Theorem 2.

Throughout the article we shall denote Lebesgue measure by $\lambda$. We begin with a lemma.

LEMMA 1. If $f$ is continuous and of bounded variation on $[0,1]$, there exists a homeomorphism $h$ of $[0,1]$ onto itself such that $f \circ h$ is a Lipschitz function.

Proof. The hypothesis implies that $f$ has finite arc length $L$. For each $x \in[0,1]$, let $A(x)$ denote the arc length of the graph of $f$ between 0 and $x$. Thus $L=A(1)$. We show the function $h$ given by $h(x)=(A(x) / L)^{-1}$ is the desired function. That $h$ is a homeomorphism of $[0,1]$ onto itslef is clear. For $x_{2}>x_{1}$ on $[0,1]$, let $t_{1}=h\left(x_{1}\right), t_{2}=h\left(x_{2}\right)$. Then

$$
\left|\frac{f \circ h\left(x_{2}\right)-f \circ h\left(x_{1}\right)}{x_{2}-x_{1}}\right|=\left|\frac{f\left(t_{2}\right)-f\left(t_{1}\right)}{h^{-1}\left(t_{2}\right)-h^{-1}\left(t_{1}\right)}\right|=L\left|\frac{f\left(t_{2}\right)-f\left(t_{1}\right)}{A\left(t_{2}\right)-A\left(t_{1}\right)}\right| \leqslant L .
$$

Thus $f \circ h$ is a Lipschitz with constant $L$.

THEOREM 1. Let $f$ be defined on $[0,1]$. A necessary and sufficient condition for there to exist a homeomorphism $h$ of $[0,1]$ onto itself, such that $f \circ h$ is

Received by the editors May 20, 1976.

AMS (MOS) subject classifications (1970). Primary 26A45.

Key words and phrases. Homeomorphism, differentiable function, bounded variation, variable monotonicity.

${ }^{1}$ Each author supported by a grant from the NSF. 
differentiable with bounded derivative, is that $f$ be continuous and of bounded variation.

Proof. The necessity of the condition is immediate. If there is an $h$ meeting the required conditions, then $f \circ h$ is in particular continuous and of bounded variation. But $f=(f \circ h) \circ h^{-1}$. Therefore, $f$ is continuous and has the same total variation as $f \circ h$.

We turn to the sufficiency of the condition. By using Lemma 1, if necessary, we may assume $f$ satisfies a Lipschitz condition with constant $L$. Let $Z=\{x: f$ is not differentiable at $x$ \}. Then $\lambda(Z)=0$. There is $X \supset Z$ such that $X$ is of type $G_{\delta}$ and $\lambda(X)=0$. By [3, Theorem 8], there exists a homeomorphism $h$, differentiable in the extended sense, of $[0,1]$ onto itself and a constant $\alpha>0$ such that

$$
\left(h^{-1}\right)^{\prime}(x)= \begin{cases}\infty & \text { if } x \in X, \\ >\alpha & \text { if } x \in[0,1] \backslash X .\end{cases}
$$

Then $h^{\prime}(x)=0$ for $x \in h^{-1}(Z)$ and $h^{\prime}(x)<1 / \alpha$ for $x \in[0,1] \backslash h^{-1}(Z)$. Let $x, y \in[0,1]$. Then

$$
\frac{(f \circ h)(y)-(f \circ h)(x)}{y-x}=\frac{f(h(y))-f(h(x))}{h(y)-h(x)} \cdot \frac{h(y)-h(x)}{y-x} .
$$

If $h(x) \in Z$, the second factor on the right side of the equation approaches zero and the first factor is bounded. Hence $(f \circ h)^{\prime}(x)=0$. If $h(x) \notin Z, f$ is differentiable at $h(x)$. So, $(f \circ h)^{\prime}(x)=f^{\prime}(h(x)) h^{\prime}(x)$ and $\left|(f \circ h)^{\prime}(x)\right| \leqslant L / \alpha$. In either case, $f \circ h$ is differentiable and its derivative is bounded in absolute value by $L / \alpha$.

According to Theorem 1, the Cantor function $f$ can be transformed into a function $g$ with a bounded derivative. The function $g$ is also a Cantor-like function relative to some nowhere dense perfect set $K$. Since $g$ is differentiable, $\lambda(K)>0$. This follows from a theorem in [1] according to which a nowhere dense perfect set $K$ can support a differentiable Cantor-like function $g$ if and only if $\lambda(K \cap I)>0$ for every open interval $I$ containing points of $K$. Theorem 1 completes this result by showing that each Cantor-like function can be transformed into a differentiable one $g$, so the countable set of values $g$ takes on the complement of its perfect set of support can be any countable dense set.

It is clear that no Cantor-like function $g$ can be continuously differentiable. To see this we need only note that $g^{\prime}$ would have to be zero on the dense set complementary to $K$, but there must be points of $K$ where $g^{\prime}>0$. The difficulty lies in the fact that $\lambda(g(K))>0$. This simple observation may be taken as the motivation for Theorem 2 , below, characterizing those functions which may be transformed into continuously differentiable ones. To make this more precise, we give a definition.

Definition. Let $f$ be defined on $[0,1]$. A point $x \in[0,1]$ is called a point of 
varying monotonicity of $f$ if there is no neighborhood of $x$ on which $f$ is either strictly monotonic or strictly constant.

Thus, for a Cantor-like function, its perfect set of support coincides with its set of points of varying monotonicity. It is immediate from the definition that the set of points of varying monotonicity of a functions is closed.

THEOREM 2. Let $f$ be defined on $[0,1]$ and let $K$ be its set of points of varying montonicity. A necessary and sufficient condition for there to exist a homeomorphism $h$ of $[0,1]$ onto $[0,1]$ such that $f \circ h$ be continuously differentiable is that $f$ be continuous and of bounded variation and that $\lambda(f(K))=0$.

It is convenient to first prove two lemmas.

LEMMA 2. If $f$ is continuous on $I=[a, b], k>0$, and $V(f, I)<k \lambda(I)$, and if the set of points of varying monotonicity of $f$ is countable, then there exists a homeomorphism $\phi$ of I onto I such that $f \circ \phi^{-1}$ is continuously differentiable on I, $\left|\left(f \circ \phi^{-1}\right)^{\prime}\right|<4 k$ on $I$ and $\left(f \circ \phi^{-1}\right)^{\prime}$ vanishes at the end points of $I$.

Proof. We affect a preliminary homeomorphism to make $f$ linear, with slope less than $k$ in magnitude, on each interval of strict monotonicity or strict constancy of $f$. We now define a positive extended real valued $g$ on $I$, which is $+\infty$ at every point of the countable set $K$ and at the end points of $I$, with $\int_{a}^{b} g(t) d t=b-a$. Since $K$ is closed and of measure zero, we may cover $K \cup\{a\} \cup\{b\}$ by a nest, $\left\{G_{n}\right\}$, of sets, each consisting of finitely many open intervals, such that $\bar{G}_{n+1} \subset G_{n}$ and $\lambda\left(G_{n}\right)<(b-a) / 2^{n+1}, n=1,2, \ldots$

Let $g_{1}$ be a continuous function such that $g_{1}(t)=1$ for $t \in \bar{G}_{1}, \frac{1}{4}<g_{1}(t)$ $\leqslant 1$ for $t \notin \bar{G}_{1}$, and $\int_{a}^{b} g_{1}(t) d t=\frac{1}{2}(b-a)$. For each $n>1$, there exist a constant $c_{n}>\frac{1}{2}$ and a continuous function $g_{n}$ such that $g_{n}(t)=c_{n}$ for $t \in \bar{G}_{n}, 0<g_{n}(t)<c_{n}$ for $t \in G_{n-1} \backslash \bar{G}_{n}, g_{n}(t)=0$ for $t \in I \backslash G_{n-1}$, and such that $\int_{a}^{b} g_{n}(t) d t=2^{-n}(b-a)$. Let $g=\sum_{1}^{\infty} g_{n}$. It is easy to verify that $g(t)>\frac{1}{4}$ for every $t \in[a, b], g(t)=+\infty$ for $t \in K \cup\{a\} \cup\{b\}, g$ is continuous in the extended sense and $\int_{a}^{b} g(t) d t=b-a$. Let $\phi(x)=a+\int_{a}^{x} g(t) d t$.

The function $\phi^{-1}$ is continuously differentiable, has derivative zero on $E=\phi(K) \cup\{a\} \cup\{b\}$ and $\left(\phi^{-1}\right)^{\prime}<4$ at every point of $I$. We show $f \circ \phi^{-1}$ is continuously differentiable on $I$ with vanishing derivative at the end points. On every interval complementary to $E$, both $f$ and $\phi^{-1}$ are continuously differentiable, so the same is true for $f \circ \phi^{-1}$. Let $x \in E$. Let $\eta>0$. There is an $n$ such that $\left(\phi^{-1}\right)^{\prime}(y)<\eta$ for every $y \in G_{n}$. Let $J \subset G_{n}, x \in J$. Then $y, z \in J$ implies $\left|\left(f \circ \phi^{-1}(y)-f \circ \phi^{-1}(z)\right) /(y-z)\right|<k \eta$. So there is $\delta>0$ such that $|x-y|<\delta$ implies $\left|\left(f \circ \phi^{-1}\right)^{\prime}(y)\right|<k \eta$. It follows that

$$
\left(f \circ \phi^{-1}\right)^{\prime}(x)=0
$$

and that $\left(f \circ \phi^{-1}\right)^{\prime}$ is continuous at $x$.

We note that the preceding proof was possible because $\lambda(K)=0$. Such a proof would not be possible for Theorem 2 because there would be no assurance that the preliminary homeomorphism transforming $f$ into a Lip- 
schitz function would carry $K$ into a zero measure set, and the function $g$ appearing in Lemma 2 could not then be constructed.

The set $K$ is the union of a perfect set $P$ and a countable subset of $K$. In the proof of Theorem 2 we shall work with the sequence $I_{1}, I_{2}, \ldots$ of open intervals complementary to $P$. Each of these intervals meets $K$ in a countable set. In our construction, at each stage we shall pick some of the $I_{k}$, contained in some interval $J$ obtained at that stage by the construction. In order to assure that we use all intervals from $\left\{I_{n}\right\}$, at each stage we pick among others the first interval in the ordering $I_{1}, I_{2}, \ldots$, on which $f$ is constant, which is contained in $J$.

The proof of Theorem 2 requires the following lemma. Suppose $f$ is continuous and of bounded variation.

Lemma 3. Suppose $\lambda(f(K))=0$. Let $J$ contain infinitely many of $I_{1}, I_{2}, \ldots$ If $V(f, J)<\varepsilon \lambda(J)$, then there are $I^{1}, \ldots, I^{N} \subset J$ from among $I_{1}, I_{2}, \ldots$ and a homeomorphism $h$ of $J$ such that $V\left(f \circ h^{-1}, h\left(I^{k}\right)\right)<2 \varepsilon \lambda\left(h\left(I^{k}\right)\right)$ for all $k$ and $V\left(f \circ h^{-1}, h\left(J_{k}\right)\right)<(\varepsilon / 2) \lambda\left(h\left(J_{k}\right)\right)$ for all $k$ where the $J_{k}$ are the intervals of $J$ complementary to the $I^{k}$.

Proof. We may assume $\lambda(K)=0$ by making a preliminary homeomorphism if necessary. Since $\lambda(f(K))=0, V(f, J)=\Sigma_{1}^{\infty} V\left(f, I^{k}\right)$ where the $I^{k}$ are the $I_{k}$ contained in $J$. There is an $N$ such that

$$
\varepsilon \sum_{1}^{N} \lambda\left(I^{k}\right)>\sum_{1}^{N} V\left(f, I^{k}\right)>\frac{7}{8} V(f, J) .
$$

Then

$$
\sum_{1}^{N+1} V\left(f, J_{k}\right)<\frac{1}{8} V(f, J)<\frac{\varepsilon}{8} \lambda(J)
$$

We first show that there is a homeomorphism $h_{N}$ of $J$ onto $J$ such that $V\left(f \circ h_{N}^{-1}, h_{N}\left(I^{k}\right)\right)<\varepsilon \lambda\left(h_{N}\left(I^{k}\right)\right)$ for each $k$. Let $\delta$ be defined by $\sum_{1}^{N} V\left(f, I^{k}\right)$ $=\delta \sum_{1}^{N} \lambda\left(I^{k}\right)$. Then $\delta<\varepsilon$. Choose a homeomorphism $h_{1}$ of $J$ onto $J$ such that $\sum_{1}^{N} \lambda\left(I^{k}\right)=\sum_{1}^{N} \lambda\left(h_{1}\left(I^{k}\right)\right)$ and $V\left(f \circ h_{1}^{-1}, h_{1}\left(I^{1}\right)\right)=\delta \lambda\left(h_{1}\left(I^{1}\right)\right)$. We proceed inductively, obtaining homeomorphisms $h_{1}, \ldots, h_{N}$ mapping $J$ onto $J$ such that for each $j=1, \ldots, N, h_{j}\left(I^{k}\right)=h_{k}\left(I^{k}\right)$ for all $k \leqslant j$,

$$
\sum_{1}^{N} \lambda\left(I^{k}\right)=\sum_{1}^{N} \lambda\left(h_{j}\left(I^{k}\right)\right)
$$

and $V\left(f \circ h_{j}^{-1}, h_{j}\left(I^{j}\right)\right)=\delta \lambda\left(h_{j}\left(I^{j}\right)\right)$. The homeomorphism $h_{N}$ has the desired property.

Now, let $\bar{h}$ be a homeomorphism of $J$ onto $J$ such that $\lambda\left(\bar{h} \circ h_{N}\left(I^{k}\right)\right)$ $=\frac{1}{2} \lambda\left(h_{N}\left(I^{k}\right)\right)$ for each $k$. Then

$$
V\left(f \circ\left(\bar{h} \circ h_{N}\right)^{-1}, \bar{h} \circ h_{N}\left(I^{k}\right)\right)<2 \varepsilon \lambda\left(\bar{h} \circ h_{N}\left(I^{k}\right)\right)
$$


and

$$
\sum_{1}^{N+1} \lambda\left(\bar{h} \circ h_{N}\left(J_{k}\right)\right)>\frac{1}{2} \lambda(J) .
$$

So

$$
\begin{aligned}
\sum_{1}^{N+1} V\left(f \circ\left(\bar{h} \circ h_{N}\right)^{-1}, \bar{h} \circ h_{N}\left(J_{k}\right)\right) & =\sum_{1}^{N+1} V\left(f, J_{k}\right)<\frac{\varepsilon}{8} \lambda(J) \\
& <\frac{\varepsilon}{4} \sum_{1}^{N+1} \lambda\left(\bar{h} \circ h_{N}\left(J_{k}\right)\right) .
\end{aligned}
$$

Allocating proportionately as in the above induction, and translating the intervals $\bar{h} \circ h_{N}\left(I^{k}\right), k=1, \ldots, N$, we obtain a homeomorphism $g$ of $J$ onto $J$ such that for $h=g \circ \bar{h} \circ h_{N}$ we have

$$
V\left(f \circ h^{-1}, h\left(J_{k}\right)\right)<(\varepsilon / 2) \lambda\left(h\left(J_{k}\right)\right) .
$$

Since $g$ only translated the intervals $\bar{h} \circ h_{N}\left(I^{k}\right)$ the inequality (1) becomes

$$
V\left(f \circ h^{-1}, h\left(I^{k}\right)\right)<2 \varepsilon \lambda\left(h\left(I^{k}\right)\right) .
$$

It follows by (2) and (3) that $h$ is the desired homeomorphism.

REMARK. We note that by requiring that $\Sigma_{1}^{N} \lambda\left(I^{k}\right)>\frac{1}{2} \lambda(J)$, we can also insure that $\sum_{1}^{N+1} \lambda\left(J^{k}\right)<\frac{3}{4} \lambda(J)$. We shall use this remark in the proof of Theorem 2 below.

\section{Proof of Theorem 2.}

Necessity. It is clear that $f$ must be continuous and of bounded variation because these properties are preserved by homeomorphic changes of variables and continuously differentiable functions have these properties. Suppose $h$ is a homeomorphism of $[0,1]$ onto itself and the function $g=f \circ h$ is continuously differentiable. The set $K$ is compact so the same is true for the set $h^{-1}(K)$. Thus the set $g \circ h^{-1}(K)=f(K)$ is measurable. If this set has positive measure there is $x_{0} \in h^{-1}(K)$ such that $g^{\prime}\left(x_{0}\right) \neq 0\left[2\right.$, p. 227]. But $g^{\prime}$ is continuous so that $g^{\prime} \neq 0$ in some neighborhood of $x_{0}$. This implies $g$ is monotonic in some neighborhood of $x_{0}$ and so $f$ is monotonic in some neighborhood of $h\left(x_{0}\right)$, a point of $K$. But this violates the definition of $K$. Thus, $\lambda(f(K))=0$ and the necessity of the condition is proved.

Sufficiency. Let $T$ be the total variation of $f$ on $[0,1]$ and let $I_{1}, I_{2}, \ldots$ be the sequence of open intervals contiguous to $P$, the perfect kernel of $K$. The sequence $\left\{I_{k}\right\}$ either has one member or is infinite. We assume it is infinite. Choose $N$ so that $\sum_{i}^{N} V\left(f, I_{k}\right)>T-\frac{1}{2}$. We apply a preliminary homeomorphism $h_{1}$ so that $V\left(f, J_{k}\right)<\frac{1}{2} \lambda\left(J_{k}\right)$ for each $k$. Here, $J_{1}, \ldots, J_{N+1}$ are the intervals complementary to $h_{1}\left(I_{1}\right), \ldots, h_{1}\left(I_{N}\right)$. The above $I_{1}, \ldots, I_{N}$ will be called of rank 1. We define a homeomorphism $h_{2}$ of rank 2 which keeps $h_{1}\left(I_{1}\right), \ldots, h_{1}\left(I_{N}\right)$ fixed and acts on $J_{1}, \ldots, J_{N+1}$. For each $j=1, \ldots, N+1$, 
by Lemma 3 , there are $I_{2, n_{j}}, \ldots, I_{2, m_{j}} \subset J_{j}$, from among $h_{1}\left(I_{1}\right), h_{1}\left(I_{2}\right), \ldots$, and a homeomorphism $h_{2}$ of $J_{j}$ onto $J_{j}$ such that

$$
V\left(f \circ h_{2}^{-1}, h_{2}\left(I_{2, k}\right)\right)<1 \cdot \lambda\left(h_{2}\left(I_{2, k}\right)\right) \text { for all } k,
$$

and

$$
V\left(f \circ h_{2}^{-1}, h_{2}\left(J_{2, k}\right)\right)<\lambda\left(h_{2}\left(J_{2, k}\right)\right) / 4 \text { for all } k,
$$

where $\left\{J_{2, k}\right\}$ is the sequence of intervals complementary to the finite sequence $\left\{I_{2, k}\right\}$ in $J_{j}$. The intervals of rank 2 are the $I_{2, k}$ and $J_{2, k}$.

We define a homeomorphism $h_{3}$ of rank 3 which keeps each $h_{2}\left(I_{2, k}\right)$ of rank 2 and each $h_{1}\left(I_{k}\right)$ of rank $I$ fixed and acts on the $J_{2, k}$. For each $k$ there are $I_{3, r_{k}}, \ldots, I_{3, s_{k}} \subset J_{2, k}$ from among $h_{2} \circ h_{1}\left(I_{1}\right), h_{2} \circ h_{1}\left(I_{2}\right), \ldots$ and a homeomorphism $h_{3}$ of $J_{2, k}$ onto $J_{2, k}$ such that

$$
V\left(f \circ h_{3}^{-1}, h_{3}\left(I_{3, j}\right)\right)<\lambda\left(h_{3}\left(I_{3, j}\right)\right) / 2 \text { for all } j,
$$

and

$$
V\left(f \circ h_{3}^{-1}, h_{3}\left(J_{3, j}\right)\right)<\lambda\left(h_{3}\left(J_{3, j}\right)\right) / 8 \text { for all } j .
$$

In this way, a rank $n=n(k)$ is associated with each $I_{k}, n$ being the first number such that $h_{m} \circ h_{m-1} \circ \cdots \circ h_{1}\left(I_{k}\right)=h_{m-1} \circ \cdots \circ h_{1}\left(I_{k}\right)$ for all $m$ $\geqslant n$. Then, if $g_{n}=h_{n} \circ h_{n-1} \circ \cdots \circ h_{1}$,

$$
(*)_{n} \quad V\left(f \circ g_{n}^{-1}, g_{n}\left(I_{k}\right)\right)<\lambda\left(g\left(I_{k}\right)\right) / 2^{n-2}, \quad n=n(k), k=1,2, \ldots
$$

By the remark following Lemma 3, we see that the construction also has the property

$$
\lim _{n}\left[\max _{k} \lambda\left(J_{n, k}\right)\right]=0 .
$$

We now define a homeomorphism $g$ of $[0,1]$ onto $[0,1]$ by $g(x)$ $=\lim _{n} g_{n}(x)$. Then, for $x \in I_{k}, g(x)=g_{n}(x)$, where $n=n(k)$, and for $x$ $\notin \cup_{k} I_{k}, g(x)$ is obtained by continuity. This is possible because $\cup_{k} I_{k}$ is dense and $\cup_{k} g\left(I_{k}\right)$ is dense because of (4).

We now modify $g$ to obtain our final homeomorphism $h$. Each $I_{k}$ has a rank $n$, so that by $(*)_{n}$,

$$
V\left(f \circ g^{-1}, g\left(I_{k}\right)\right)<\lambda\left(g\left(I_{k}\right)\right) / 2^{n-2} .
$$

Since $f \circ g^{-1}$ is either strictly increasing, decreasing, or constant on $g\left(I_{k}\right)$, or there is a "partitioning" of $g\left(I_{k}\right)$ into countably many intervals on each of which $f \circ g^{-1}$ is as above there is, by Lemma 2, a homeomorphism $\phi$ of $g\left(I_{k}\right)$ onto $g\left(I_{k}\right)$ such that $f \circ g^{-1} \circ \phi^{-1}$ is continuously differentiable on $\phi \circ g\left(I_{k}\right)$ $=g\left(I_{k}\right)$, has derivative smaller in absolute value than $1 / 2^{n-2}$ at each point of 
the interval, and the derivative converges to zero at the end points. Let $h=\phi \circ g$. Then $f \circ h^{-1}$ is continuously differentiable on $[0,1]$. For this we need only show that the derivative is zero at each point of $h(P)$ when taken relative to the complement of $h(P)$. Let $x \in h(P)$. Let $\varepsilon>0$. There is an $n$ such that $1 / 2^{n-1}<\varepsilon$. There is a $\delta>0$ such that $(x-\delta, x+\delta)$ meets only intervals $h\left(I_{k}\right)$ for which the rank of $I_{k}$ exceeds $n$. If $y \in h\left(I_{k}\right)$ and $|x-y|<\delta$ then, noticing that $f \circ h^{-1}$ is absolutely continuous,

$$
\left|f \circ h^{-1}(y)-f \circ h^{-1}(x)\right| \leqslant \int_{x}^{y}\left|\left(f \circ h^{-1}\right)(t)\right| d t \leqslant \varepsilon|x-y| .
$$

So the derivative of $f \circ h^{-1}$ is zero at $x$.

REMARK. It is necessary that $f$ be in $V B G_{*}$ in order for there to be a homeomorphism $h$ such that $f \circ h^{-1}$ is everywhere finitely differentiable. Fleissner and Foran have advised us that the sufficiency follows easily from Theorem 1 and a result of Tolstov in Mat. Sb. 53 (95) (1961), pp. 387-392.

\section{REFERENCES}

1. A. M. Bruckner and John. L. Leonard, On differentiable functions having an everywhere dense set of intervals of constancy, Canad. Math. Bull. 8 (1965), 73-76. MR 30 \#4870.

2. S. Saks, Theory of the integral, Monografie Mat., Warsaw, 1933; rev. ed., English transl., Stechert, New York, 1937.

3. Z. Zahorski, Sur la première dérivée, Trans. Amer. Math. Soc. 69 (1950), 1-54. MR 12, 247.

Department of Mathematics, University of California at Santa Barbara, Santa Barbara, California 93106

Department of Mathematics, Purdue University, West Lafayette, Indiana 47907 